\title{
Coronary CT Angiography versus Standard Evaluation in Acute Chest Pain
}

Udo Hoffmann, M.D., M.P.H., Quynh A. Truong, M.D., M.P.H., David A. Schoenfeld, Ph.D., Eric T. Chou, M.D., Pamela K. Woodard, M.D., John T. Nagurney, M.D., M.P.H., J. Hector Pope, M.D., Thomas H. Hauser, M.D., M.P.H., Charles S. White, M.D., Scott G. Weiner, M.D., M.P.H., Shant Kalanjian, M.D., Michael E. Mullins, M.D., Issam Mikati, M.D., W. Frank Peacock, M.D., Pearl Zakroysky, B.A., Douglas Hayden, Ph.D., Alexander Goehler, M.D., Ph.D., Hang Lee, Ph.D., G. Scott Gazelle, M.D., M.P.H., Ph.D., Stephen D. Wiviott, M.D., Jerome L. Fleg, M.D., and James E. Udelson, M.D. for the ROMICAT-II Investigators

\section{Abstract}

Background-It is unclear whether an evaluation incorporating coronary computed tomographic angiography (CCTA) is more effective than standard evaluation in the emergency department in patients with symptoms suggestive of acute coronary syndromes.

Methods-In this multicenter trial, we randomly assigned patients 40 to 74 years of age with symptoms suggestive of acute coronary syndromes but without ischemic electrocardiographic changes or an initial positive troponin test to early CCTA or to standard evaluation in the emergency department on weekdays during daylight hours between April 2010 and January 2012. The primary end point was length of stay in the hospital. Secondary end points included rates of discharge from the emergency department, major adverse cardiovascular events at 28 days, and cumulative costs. Safety end points were undetected acute coronary syndromes.

Results-The rate of acute coronary syndromes among 1000 patients with a mean $( \pm$ SD) age of $54 \pm 8$ years ( $47 \%$ women) was $8 \%$. After early CCTA, as compared with standard evaluation, the mean length of stay in the hospital was reduced by 7.6 hours $(\mathrm{P}<0.001)$ and more patients were discharged directly from the emergency department $(47 \%$ vs. $12 \%, \mathrm{P}<0.001)$. There were no undetected acute coronary syndromes and no significant differences in major adverse cardiovascular events at 28 days. After CCTA, there was more downstream testing and higher radiation exposure. The cumulative mean cost of care was similar in the CCTA group and the standard-evaluation group ( $\$ 4,289$ and $\$ 4,060$, respectively; $\mathrm{P}=0.65)$.

Conclusions-In patients in the emergency department with symptoms suggestive of acute coronary syndromes, incorporating CCTA into a triage strategy improved the efficiency of clinical decision making, as compared with a standard evaluation in the emergency department, but it resulted in an increase in downstream testing and radiation exposure with no decrease in the overall costs of care. (Funded by the National Heart, Lung, and Blood Institute; ROMICAT-II ClinicalTrials.gov number, NCT01084239.)

Treatment of Patients with Acute chest pain but an inconclusive initial evaluation with the use of biomarkers and electrocardiographic (ECG) testing is often diagnostically challenging

Copyright $(2012$ Massachusetts Medical Society.

Address reprint requests to Dr. Hoffmann at Massachusetts General Hospital, Cardiac MR PET CT Program, 165 Cambridge St., Suite 400, Boston, MA 02114, or at uhoffmann@ partners.org.

No other potential conflict of interest relevant to this article was reported.

Disclosure forms provided by the authors are available with the full text of this article at NEJM.org. 
and inefficient. The majority of patients with acute coronary syndromes have underlying coronary artery disease. ${ }^{1}$ Contrast-enhanced coronary computed tomographic angiography (CCTA) has high sensitivity and specificity for the detection of clinically significant coronary artery disease, as compared with invasive coronary angiography, in patients in stable condition with suspected or known coronary artery disease..$^{2-5}$

Rule Out Myocardial Infarction/Ischemia Using Computer Assisted Tomography (ROMICAT-I), ${ }^{6}$ a blinded observational study involving patients in the emergency department with suspected acute coronary syndromes, and other studies ${ }^{7,8}$ have shown that normal findings on CCTA have a very high negative predictive value for ruling out acute coronary syndromes during the index hospitalization and the occurrence of major adverse cardiovascular events over the next 2 years. ${ }^{7,9}$ The results of two previous randomized, multicenter trials ${ }^{10,11}$ suggest that CCTA may facilitate safe and earlier triage of low-risk patients and that CCTA can rule out coronary artery disease faster than stress myocardialperfusion imaging. However, imaging the coronary anatomy with CCTA can involve more procedures and greater costs than functional testing. ${ }^{12}$ Thus, equipoise exists regarding the effectiveness of incorporating CCTA into an evaluation strategy in the emergency department.

The objectives of this study were to compare the effectiveness of a CCTA-based evaluation strategy with that of standard evaluation in the emergency department for patients with symptoms suggestive of an acute coronary syndrome and to evaluate the downstream testing, cost, and radiation exposure associated with CCTA.

\section{Methods}

\section{Study Design and Oversight}

ROMICAT-II was designed as a randomized, controlled, multicenter trial in which an evaluation and management strategy that included CCTA as a first diagnostic test performed as early as possible was compared with a standard emergency department evaluation for patients with acute chest pain suggestive of an acute coronary syndrome. After random assignments had been made to initial CCTA or standard evaluation without CCTA, patient care in both groups was not mandated by the study protocol but instead was at the discretion of local physicians. The design of ROMICAT-II has been described in detail previously, ${ }^{13}$ and the study protocol is available with the full text of this article at NEJM.org.

\section{Study Population}

Patient enrollment began on April 23, 2010, and ended on January 30, 2012, at nine hospitals in the United States. All patients provided written informed consent to participate in the study. Eligibility criteria were chosen according to the ROMICAT-I study, ${ }^{6}$ with the goal of enrolling a population with a similar prevalence of acute coronary syndromes (approximately 8\%). Eligible patients were 40 to 74 years of age, presented to the emergency department with chest pain (or the anginal equivalent) of at least 5 minutes' duration within 24 hours before presentation in the emergency department, were in sinus rhythm, and warranted further risk stratification to rule out acute coronary syndromes, as determined by an attending physician in the emergency department. Major exclusion criteria were a history of known coronary artery disease, new diagnostic ischemic changes on the initial ECG, an initial troponin level in excess of the 99th percentile of the local assay, impaired renal function (creatinine level, $>1.5 \mathrm{mg}$ per deciliter [132.6 $\mu \mathrm{mol}$ per liter]), hemodynamic or clinical instability, known allergy to an iodinated contrast agent, a bodymass index (the weight in kilograms divided by the square of the height in meters) greater than 40 , or currently symptomatic asthma. 


\section{Study Protocol}

Eligible patients were identified, provided written informed consent, and were randomly assigned at their initial evaluation in the emergency department during weekday daytime hours. Patients were randomly assigned in a 1:1 ratio to either CCTA as part of the initial evaluation in the emergency department or the standard evaluation strategy in the emergency department at that site. All test results were provided to emergency department physicians in real time. Additional care was not mandated by the study protocol in either randomization group.

The discharge diagnosis was based on the local physicians' assessment. The discharge diagnoses were adjudicated separately by a clinical end-points committee in a predefined sample of 242 patients, which included all patients with acute coronary syndromes, the first 8 patients enrolled at each site, $4 \%$ of patients discharged with cardiac symptoms, and a randomly selected subgroup of $10 \%$ of all patients. The definitions of acute coronary syndromes are provided in the Supplementary Appendix, available at NEJM.org.

To ascertain potentially undetected acute coronary syndromes and as a safety measure, patients discharged within 24 hours after presentation in the emergency department were contacted by telephone within 72 hours to assess their clinical status. A follow-up telephone call to all patients was also conducted 28 days after discharge. During telephone calls, information on repeat visits to the emergency department or rehospitalizations for recurrent chest pain (including diagnostic testing, interventions, and clinical events during follow-up) was obtained and verified by the collection of medical records.

\section{CCTA}

Before the start of the study, participating sites were not routinely performing CCTA in patients in the emergency department to detect acute coronary syndromes, but they were required to use at least 64-slice CT technology for patient assessment. Protocols involving both retrospectively ECG-gated and prospectively ECG-triggered CCTA were permitted, with use according to published guidelines. ${ }^{14}$ The use of tube modulation to lower radiation exposure was strongly encouraged. CCTA images were interpreted on-site in real time, and the results were communicated to the responsible clinician.

\section{End Points}

The prespecified primary end point was the length of the hospital stay, defined as the time from presentation in the emergency department to the time of the discharge order. This end point was chosen because it reflects the summary of actions taken in response to clinical information and test results, as well as logistical, cost, and medical and legal considerations in participating centers.

Secondary effectiveness end points included the time to diagnosis, defined as the time from presentation in the emergency department until the first diagnostic test that led to the diagnosis of an acute coronary syndrome, or as the time from presentation in the emergency department to the final test that was used to rule out an acute coronary syndrome. The rate of direct discharge from the emergency department was defined as the proportion of patients discharged from the emergency department without admission to an observation unit or the hospital. Resource utilization was defined as any diagnostic testing (CCTA, exercise treadmill testing, nuclear imaging, stress echocardiography, or cardiac catheterization) or interventions from the index assessment in the emergency department to follow-up at 28 days, and it included resources used during repeat visits to the emergency department or hospitalization for recurrent chest pain. Cumulative radiation exposure was defined as radiation exposure from testing, including CCTA, nuclear perfusion imaging, and invasive 
coronary angiography, measured in millisieverts and calculated with the use of standard methods ${ }^{15}$ during the index care episode (the visit to the emergency department and hospitalization) and follow-up. Health care costs during the index care episode were assessed from reports from hospital cost-accounting systems and physician billing records and were adjusted to 2011 dollars. Mean costs for patient care, diagnostic testing, and interventions during the index care episode were used to estimate the costs during follow-up.

Safety variables prespecified as secondary end points included an undetected acute coronary syndrome (defined as an unexpected cardiovascular event within 72 hours after hospital discharge in patients with a hospital stay of $<24$ hours), to ensure that potentially earlier discharge in the CCTA group was not associated with increased adverse events, major adverse cardiovascular events (defined as death, myocardial infarction, unstable angina, or urgent coronary revascularization within 28 days), and periprocedural complications (stroke, bleeding, anaphylaxis, or renal failure). These predefined safety variables were adjudicated by an external, independent clinical-events committee.

\section{Statistical Analyses}

All statistical analyses were performed by an independent data coordinating center on the basis of an intention-to-treat analysis. Continuous data are presented as means $\pm \mathrm{SD}$ and medians with interquartile ranges. Comparisons between groups were performed with the use of an independent-sample t-test for continuous variables, Fisher's exact test for categorical variables, and the Wilcoxon rank-sum test for ordinal variables. A two-sided $\mathrm{P}$ value of less than 0.05 was considered to indicate statistical significance. Concordance between the discharge diagnosis made at the study site and the independently adjudicated diagnosis in a selected subpopulation was assessed with the use of the kappa statistic.

The study was designed to have greater than $83 \%$ power with the use of a t-test at a twosided 5\% significance level if the true between-group difference in the length of stay in the hospital was at least 8.3 hours. Details of the simulation are described elsewhere. ${ }^{13}$

The study did not have predefined stopping rules or boundaries with respect to the primary end point or safety end points. Rather, the data and safety monitoring board was responsible for assessing every case in which an acute coronary syndrome might have been undetected.

\section{Results}

\section{Study Population}

Of 1000 enrolled patients, 501 were randomly assigned to CCTA and 499 were randomly assigned to a standard evaluation in the emergency department. All patients were included in the intention-to-treat analysis (Fig. 1). CCTA was not performed in 28 patients (6\%) because of the patient's decision to decline CCTA ( 9 patients), safety concerns (5 patients), unavailability of CCTA (5 patients), or technical difficulties (9 patients). Overall, 987 of 1000 randomly assigned patients (99\%) had complete follow-up at 28 days. The original medical records for repeat visits to the emergency department or hospitalizations were available in all cases.

Baseline characteristics of the study population are shown in Table 1. After a complete evaluation, 75 patients (8\%) had a final diagnosis of an acute coronary syndrome. Agreement between the site and independent adjudication for the discharge diagnosis was very high (concordance, 98\% [236 of 242 patients]; kappa, 0.94). 


\section{Primary and Secondary Effectiveness End Points}

The effectiveness end points are shown in Table 2 . The primary end point met the prespecified criterion for significance, since the average length of the hospital stay in the group of patients randomly assigned to CCTA was decreased by 7.6 hours, as compared with the group randomly assigned to a standard emergency department evaluation $(\mathrm{P}<0.001)$. Figure 2 shows the cumulative distribution of discharged patients with length-ofstay data in the two groups. Notably, 50\% of the patients in the CCTA group were discharged within 8.6 hours after presentation, as compared with $10 \%$ of the patients randomly assigned to a standard evaluation in the emergency department. In the subgroup of patients with a final diagnosis of an acute coronary syndrome, the length of stay in the hospital was similar after CCTA and after standard evaluation in the emergency department.

In the overall cohort and also in the subgroups with or without a final diagnosis of an acute coronary syndrome, the mean time to diagnosis was significantly decreased with CCTA as compared with a standard evaluation. Patients in the CCTA group were more often directly discharged from the emergency department (47\%, vs. $12 \%$ of patients in the standardevaluation group; $\mathrm{P}<0.001$ ), with fewer admissions to an observation unit.

\section{Safety End Points}

Prespecified clinical adverse events were infrequent in this trial (Table 2). No cases of undetected acute coronary syndromes were identified in either study group. Overall, there were eight major adverse cardiovascular events during the 28-day follow-up: six after standard evaluation in the emergency department (four myocardial infarctions and two cases of unstable angina pectoris for which percutaneous coronary intervention was required) and two after CCTA (one myocardial infarction and one case of unstable angina pectoris for which percutaneous coronary intervention was required $)(P=0.18)$. In both of the latter patients, CCTA established clinically significant coronary artery disease during the index hospitalization, but both patients had negative stress tests and were initially treated medically. Two periprocedural complications occurred in the CCTA group (perioperative bleeding after cardiothoracic surgery for an identified anomalous coronary artery and a transient increase in the creatinine level after CCTA without the need for dialysis in a patient with a urethral stone and hydronephrosis), and no periprocedural complications occurred in the standard-evaluation group.

\section{Resource Utilization and Radiation Exposure}

Table 3 shows resource utilization. Overall, more diagnostic testing was performed in the CCTA group than in the standard-evaluation group $(\mathrm{P}<0.001)$. Both the cumulative rate of invasive coronary angiography during the index hospitalization and follow-up and the rate of coronary revascularization were higher among patients in the CCTA group than among patients in the standard-evaluation group, but the differences were not significant $(\mathrm{P}=0.06$ and $\mathrm{P}=0.16$, respectively) (Table 3 ).

Nearly all patients in the CCTA group (484 of 501 patients; 97\%), but only 167 of 499 patients randomly assigned to standard evaluation $(33 \%)$ received radiation exposure from an imaging test or procedure. Hence, cumulative radiation exposure was significantly higher in the CCTA group (Table 3). The mean radiation exposure from CCTA was $11.3 \pm 5.3 \mathrm{mSv}$ and was lower than that from single-photon-emission CT $(14.1 \pm 4.8 \mathrm{mSv}, \mathrm{P}<0.001)$. The 78 patients who underwent CCTA with the use of an advanced 128-slice, dual-source CT scanner had lower radiation exposure $(6.2 \pm 3.8 \mathrm{mSv})$ than did the remaining patients $(12.3 \pm 5$ $\mathrm{mSv})$. 
Detailed cost data were available in a subgroup of all 649 patients from five centers (Table

3). The mean costs of care from the initial visit in the emergency department through the 28day follow-up were similar in the CCTA group and the group that received standard evaluation in the emergency department $(\mathrm{P}=0.65)$.

\section{Discussion}

This prospective, multicenter, randomized, strategy-controlled trial was designed primarily to assess whether CCTA, incorporated early into an evaluation strategy for patients presenting to an emergency department with chest pain suggestive of an acute coronary syndrome, safely improves the efficiency of clinical decision making, as compared with a standard evaluation in the emergency department. The cumulative costs of diagnostic tests, interventions, and radiation exposure were also evaluated. The average length of stay in the hospital, the primary end point of the trial, was significantly reduced in the CCTA group, as was the time to diagnosis. Furthermore, rates of direct discharge from the emergency department were higher with CCTA than with a standard evaluation in the emergency department. These results were achieved without putting patients at greater risk for undetected acute coronary syndromes and without an increase in the cost of care. However, we observed increased diagnostic testing in the CCTA group and increased radiation exposure.

An important consideration when results show more efficient triage is whether that gain is achieved at the risk of undetected acute coronary syndromes. There were no undetected cases of acute coronary syndromes in either study group, suggesting that the earlier and greater number of discharges in the CCTA group did not result in any missed diagnoses. More major adverse cardiovascular events were observed in the standard-evaluation group than in the CCTA group, though the study did not have the statistical power to support the conclusion that major adverse cardiovascular events may be reduced after a CCTA-based evaluation.

The prevalence of acute coronary syndromes in a patient population is an important determinant of the risk-benefit ratio, given that CCTA is an advanced diagnostic imaging test that entails the administration of iodinated contrast material, radiation exposure, and costs. In our study population of patients who were at intermediate risk for acute coronary syndromes (observed rate of acute coronary syndromes, 7.5\%, vs. 2 and $4 \%$ in previous studies ${ }^{10,11}$ ), a greater number of invasive coronary procedures were performed after CCTA than after a standard evaluation. Information on the presence of anatomical coronary artery disease may influence clinical decision making toward invasive angiography. This concept is consistent with recent data suggesting that in a Medicare population, imaging of the coronary anatomy with CCTA in a nonemergency setting led to greater use of downstream testing and procedures, as compared with functional stress testing. ${ }^{12}$

In this trial, no decrease in total costs for the index visit and during 28-day follow-up was observed in a subgroup of 649 patients from five of nine sites in which complete billing data were available. Long-term outcome data are not available; such data might have allowed a determination of whether CCTA results in fewer repeat visits to the emergency department and hospitalizations over a longer time course.

Cumulative radiation exposure was higher in the group randomly assigned to CCTA than in the standard-evaluation group. Recent data show that diagnostic-quality CCTA imaging can be performed with exposure of less than $5 \mathrm{mSv}$ in selected patients; this suggests that future studies could use lower doses of radiation. ${ }^{16,17}$ Lower-dose radiation should be considered in efforts to apply this strategy more widely, as well as in particular groups of patients. 
There are several limitations of the present study and analysis. Enrollment occurred only during weekday hours when all imaging testing was available with technologists and readers on site. However, the results of triage decision making and particularly the timing of decisions to discharge or hospitalize patients would probably be different if the imaging studies were carried out during the night, when testing and interpretation are not as accessible. Similarly, the results cannot be generalized to clinical sites that perform a dedicated accelerated diagnostic protocol ${ }^{18}$ in the standard evaluation.

Inherent in the design of any randomized, comparative-effectiveness trial assessing a testing procedure is the lack of blinding to the intervention. We acknowledge that there may have been a bias in decision making toward earlier discharge in the CCTA group. For both groups of patients, however, the decision making was left to a large number of clinicians at the nine sites who were not directly associated with the study and whose decisions were subject to the same imperatives to provide high-quality clinical care and to take into account medical and legal considerations. Finally, the results of this study may not be applicable to populations that we did not study, including patients younger than 40 years of age and those older than 74 years of age.

In conclusion, in this trial involving patients with suspected acute coronary syndromes, an evaluation strategy incorporating early CCTA, as compared with a standard evaluation strategy, improved the efficiency of clinical decision making for triage in the emergency department, with a shorter length of stay in the hospital and more direct discharges from the emergency department. This improvement appeared to be accomplished safely, without putting patients at greater risk for undetected acute coronary syndromes. There was increased diagnostic testing and higher radiation exposure in the CCTA group, with no overall reduction in the cost of care. These data should allow providers and patients to make informed decisions about the use of this technology as an option for evaluation when symptoms are suggestive of an acute coronary syndrome.

\section{Supplementary Material}

Refer to Web version on PubMed Central for supplementary material.

\section{Acknowledgments}

Supported by grants from the National Heart, Lung, and Blood Institute (U01HL092040 and U01HL092022) and the National Institutes of Health (UL1RR025758, K23HL098370, and L30HL093896, to Dr. Truong).

Dr. Gazelle reports receiving consulting fees from GE Healthcare; Dr. Hauser, receiving consulting fees from Astellas and the Harvard Clinical Research Institute; Dr. Hoffmann, receiving grant support from the American College of Radiology Imaging Network, Bracco Diagnostics, Genentech, and Siemens Healthcare on behalf of his institution; Dr. Nagurney, receiving grant support from Alere (Biosite), Brahms Diagnostica (Fischer), and Nanosphere on behalf of his institution; Dr. Truong, receiving grant support from St. Jude Medical and Qi Imaging on behalf of her institution and travel support from Medconvent and the Society of Cardiac Computed Tomography; Dr. Wiviott, receiving consulting fees from Arena Pharmaceuticals, AstraZeneca, Bayer, BristolMyers Squibb, and Ortho-McNeil, grant support from AstraZeneca, Daiichi Sankyo, Eli Lilly, and Merck and Schering-Plough on behalf of his institution, and lecture fees from Astra-Zeneca, Daiichi Sankyo, Eli Lilly, Novartis, and Schering-Plough; and Dr. Udelson, being on the scientific advisory board of Lantheus Medical Imaging.

\section{Appendix}

\section{Appendix 1:}

The author's affiliations are as follows: the Department of Radiology (U.H., P.Z., A.G., G.S.G.), the Cardiac MR PET CT Program (U.H., Q.A.T., P.Z., A.G.), the Division of 
Cardiology (U.H., Q.A.T.), the Department of Emergency Medicine (J.T.N.), the Biostatistics Center (D.A.S., D.H., H.L.), and the Institute of Technology Assessment (A.G., G.S.G.), Massachusetts General Hospital, Boston; Harvard Medical School, Boston (U.H., Q.A.T., D.A.S., T.H.H., A.G., H.L., G.S.G., S.D.W.); the Department of Emergency Medicine, Baystate Medical Center, Springfield, MA (J.H.P.); the Cardiovascular Institute, Beth Israel Deaconess Medical Center, Boston (T.H.H.); the Department of Emergency Medicine (S.G.W.) and the Division of Cardiology and the CardioVascular Center (J.E.U.), Tufts Medical Center, Boston; and the Cardiovascular Division, Brigham and Women's Hospital, Boston (S.D.W.); Kaiser Permanente Fontana Medical Center, Fontana, CA (E.T.C., S.K.); Mallinckrodt Institute of Radiology (P.K.W.) and the Division of Emergency Medicine (M.E.M.), Washington University School of Medicine, St. Louis; University of Maryland School of Medicine, Baltimore (C.S.W.); the Division of Cardiovascular Sciences, National Heart, Lung, and Blood Institute, Bethesda, MD (J.L.F.); the Department of Emergency Medicine, Department of Medicine, Center for Cardiovascular Innovation, Northwestern University Feinberg School of Medicine, Chicago (I.M.); the Department of Emergency Medicine, Baylor College of Medicine, Houston (W.F.P.); and the Thrombolysis in Myocardial Infarction (TIMI) Study Group, Boston (S.D.W.).

\section{References}

1. Roe MT, Harrington RA, Prosper DM, et al. Clinical and therapeutic profile of patients presenting with acute coronary syndromes who do not have significant coronary artery disease. Circulation. 2000; 102:1101-6. [PubMed: 10973837]

2. Miller JM, Rochitte CE, Dewey M, et al. Diagnostic performance of coronary angiography by 64row CT. N Engl J Med. 2008; 359:2324-36. [PubMed: 19038879]

3. Budoff MJ, Dowe D, Jollis JG, et al. Diagnostic performance of 64-multidetector row coronary computed tomographic angiography for evaluation of coronary artery stenosis in individuals without known coronary artery disease: results from the prospective multicenter ACCURACY (Assessment by Coronary Computed Tomographic Angiography of Individuals Undergoing Invasive Coronary Angiography) trial. J Am Coll Cardiol. 2008; 52:1724-32. [PubMed: 19007693]

4. Marano R, De Cobelli F, Floriani I, et al. Italian multicenter, prospective study to evaluate the negative predictive value of 16- and 64-slice MDCT imaging in patients scheduled for coronary angiography (NIMISCAD-Non Invasive Multicenter Italian Study for Coronary Artery Disease). Eur Radiol. 2009; 19:1114-23. [PubMed: 19089430]

5. Meijboom WB, Meijs MF, Schuijf JD, et al. Diagnostic accuracy of 64-slice computed tomography coronary angiography: a prospective, multicenter, multivendor study. J Am Coll Cardiol. 2008; 52:2135-44. [PubMed: 19095130]

6. Hoffmann U, Bamberg F, Chae CU, et al. Coronary computed tomography angiography for early triage of patients with acute chest pain: the ROMICAT (Rule Out Myocardial Infarction using Computer Assisted Tomography) trial. J Am Coll Cardiol. 2009; 53:1642-50. [PubMed: 19406338]

7. Hollander JE, Chang AM, Shofer FS, et al. One-year outcomes following coronary computerized tomographic angiography for evaluation of emergency department patients with potential acute coronary syndrome. Acad Emerg Med. 2009; 16:693-8. [PubMed: 19594460]

8. Rubinshtein R, Halon DA, Gaspar T, et al. Usefulness of 64-slice cardiac computed tomographic angiography for diagnosing acute coronary syndromes and predicting clinical outcome in emergency department patients with chest pain of uncertain origin. Circulation. 2007; 115:1762-8. [PubMed: 17372178]

9. Schlett CL, Banerji D, Siegel E, et al. Prognostic value of CT angiography for major adverse cardiac events in patients with acute chest pain from the emergency department: 2-year outcomes of the ROMICAT trial. JACC Cardiovasc Imaging. 2011; 4:481-91. [PubMed: 21565735]

10. Goldstein JA, Chinnaiyan KM, Abidov A, et al. The CT-STAT (Coronary Computed Tomographic Angiography for Systematic Triage of Acute Chest Pain Patients to Treatment) trial. J Am Coll Cardiol. 2011; 58:1414-22. [PubMed: 21939822] 
11. Litt HI, Gatsonis C, Snyder B, et al. CT angiography for safe discharge of patients with possible acute coronary syndromes. N Engl J Med. 2012; 366:1393-403. [PubMed: 22449295]

12. Shreibati JB, Baker LC, Hlatky MA. Association of coronary CT angiography or stress testing with subsequent utilization and spending among Medicare beneficiaries. JAMA. 2011; 306:2128-36. [PubMed: 22089720]

13. Hoffmann U, Truong QA, Fleg JL, et al. Design of the Rule Out Myocardial Ischemia/Infarction Using Computer Assisted Tomography: a multicenter randomized comparative effectiveness trial of cardiac computed tomography versus alternative triage strategies in patients with acute chest pain in the emergency department. Am Heart J. 2012; 163:330-8. [PubMed: 22424002]

14. Abbara S, Arbab-Zadeh A, Callister TQ, et al. SCCT guidelines for performance of coronary computed tomographic angiography: a report of the Society of Cardiovascular Computed Tomography Guidelines Committee. J Cardiovasc Comput Tomogr. 2009; 3:190-204. [PubMed: 19409872]

15. Gerber TC, Carr JJ, Arai AE, et al. Ionizing radiation in cardiac imaging: a science advisory from the American Heart Association Committee on Cardiac Imaging of the Council on Clinical Cardiology and Committee on Cardiovascular Imaging and Intervention of the Council on Cardiovascular Radiology and Intervention. Circulation. 2009; 119:1056-65. [PubMed: 19188512]

16. von Ballmoos MW, Haring B, Juillerat P, Alkadhi H. Meta-analysis: diagnostic performance of low-radiation-dose coronary computed tomography angiography. Ann Intern Med. 2011; 154:41320. Erratum, Ann Intern Med 2011;154:848. [PubMed: 21403076]

17. Achenbach S, Marwan M, Ropers D, et al. Coronary computed tomography angiography with a consistent dose below $1 \mathrm{mSv}$ using prospectively electrocardiogram-triggered high-pitch spiral acquisition. Eur Heart J. 2010; 31:340-6. [PubMed: 19897497]

18. Than M, Cullen L, Reid CM, et al. A 2-h diagnostic protocol to assess patients with chest pain symptoms in the Asia-Pacific region (ASPECT): a prospective observational validation study. Lancet. 2011; 377:1077-84. [PubMed: 21435709] 


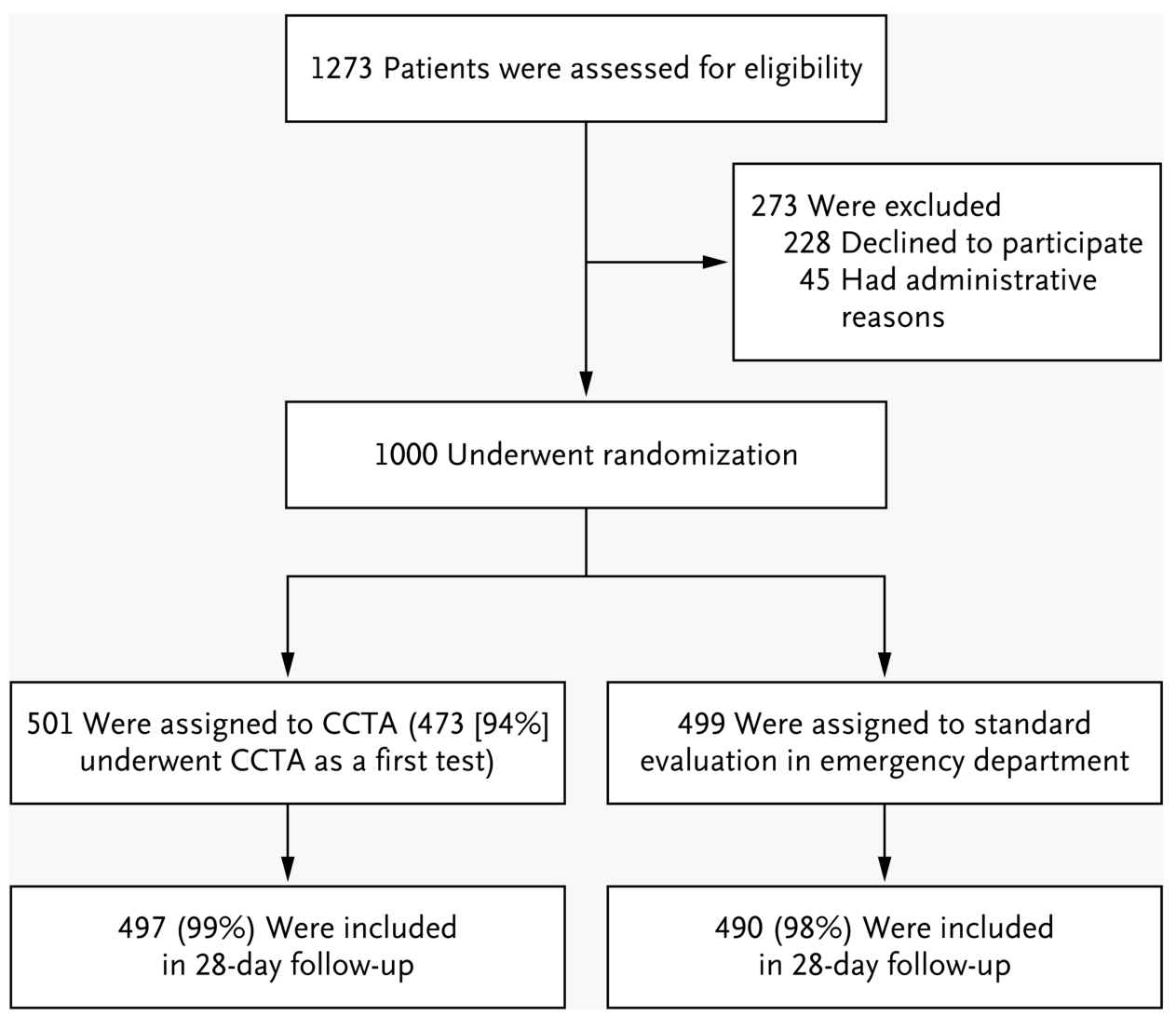

Figure 1. Screening, Randomization, and Follow-up of the Study Patients CCTA denotes coronary computed tomographic angiography. 


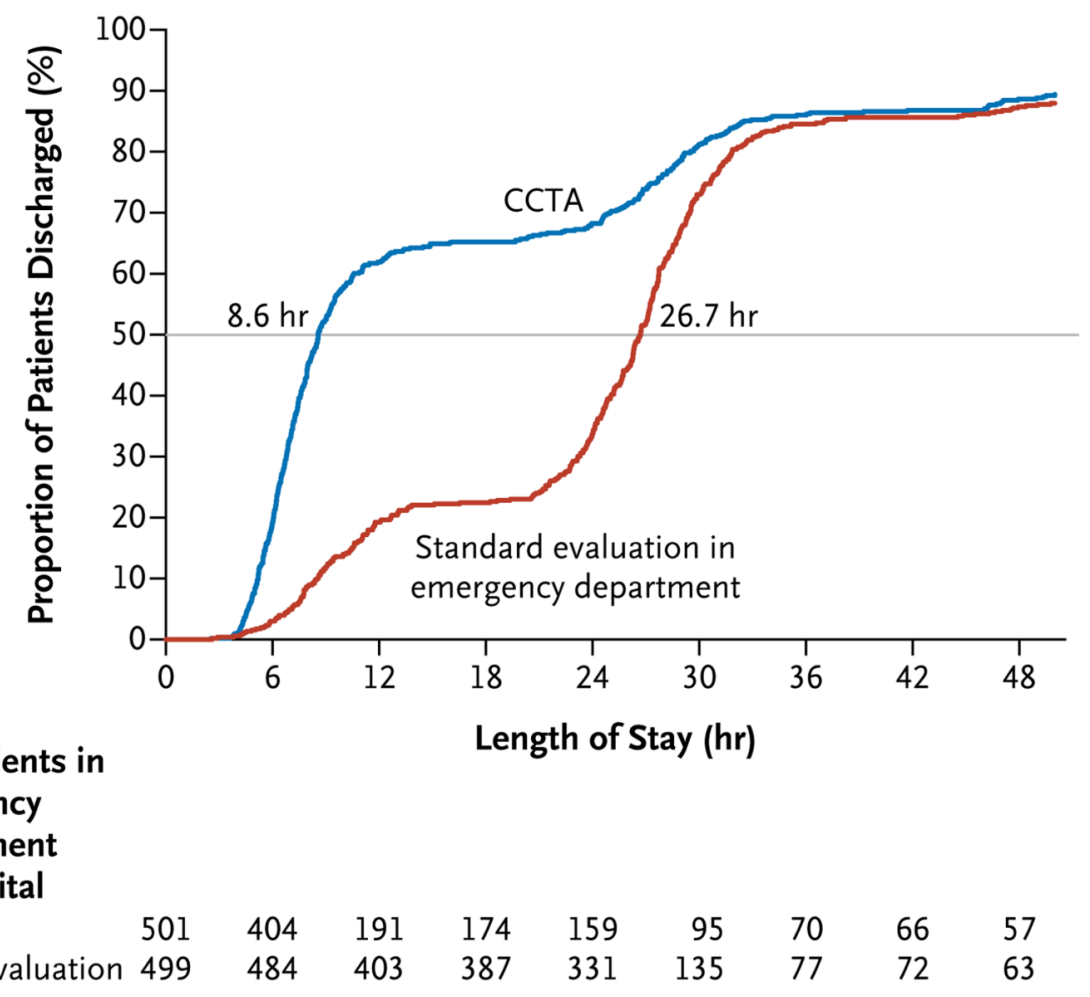

\section{No. of Patients in \\ Emergency Department or Hospital}

$\begin{array}{lrrrrrrrrr}\text { CCTA } & 501 & 404 & 191 & 174 & 159 & 95 & 70 & 66 & 57 \\ \text { Standard evaluation } & 499 & 484 & 403 & 387 & 331 & 135 & 77 & 72 & 63\end{array}$

Figure 2. Length of Stay in the Hospital and Proportion of Patients Discharged The cumulative frequency of discharge from the index visit according to the length of stay is shown. The horizontal line indicates the median length of stay in the two study groups, which was significantly different (8.6 hours in the CCTA group vs. 26.7 hours in the standard-evaluation group, $\mathrm{P}<0.001)$. 
Table 1

Baseline Demographic and Clinical Characteristics of the Patients.

\begin{tabular}{|c|c|c|c|}
\hline Variable & CCTA $(N=501)$ & $\begin{array}{l}\text { Standard Evaluation }(\mathrm{N} \\
\quad=499)\end{array}$ & P Value \\
\hline Mean age - yr & $54 \pm 8$ & $54 \pm 8$ & 0.44 \\
\hline Female sex $-\%$ & 48 & 46 & 0.57 \\
\hline \multicolumn{4}{|l|}{ Race or ethnic group - no. (\%) } \\
\hline Black & $141(28)$ & $141(28)$ & 1.00 \\
\hline White & $330(66)$ & $330(66)$ & 0.95 \\
\hline Asian & $18(4)$ & $13(23)$ & 0.47 \\
\hline Other & $12(2)$ & $18(4)$ & 0.27 \\
\hline Non-Hispanic & $435(87)$ & $422(85)$ & 0.57 \\
\hline \multicolumn{4}{|l|}{ Cardiovascular risk factors - no. $(\%)$} \\
\hline Hypertension & $269(54)$ & $272(54)$ & 0.80 \\
\hline Diabetes mellitus & $86(17)$ & $87(17)$ & 0.93 \\
\hline Dyslipidemia & $230(46)$ & $224(45)$ & 0.75 \\
\hline Former or current smoker & $249(50)$ & $243(49)$ & 0.75 \\
\hline Family history of premature coronary artery disease & $135(27)$ & $136(27)$ & 0.94 \\
\hline No. of cardiovascular risk factors $-\%$ & & & 0.68 \\
\hline 0 or 1 & 36 & 38 & \\
\hline 2 or 3 & 54 & 52 & \\
\hline$\geq 4$ & 10 & 10 & \\
\hline \multicolumn{4}{|l|}{ Relevant prior medication — no. (\%) } \\
\hline Aspirin & $115(23)$ & $113(23)$ & 0.94 \\
\hline Beta-blocker & $88(18)$ & $82(16)$ & 0.67 \\
\hline Statin & $143(28)$ & $151(30)$ & 0.58 \\
\hline \multicolumn{4}{|l|}{ Initial presentation in emergency department } \\
\hline Chief symptom — no. $(\%)$ & & & 0.47 \\
\hline Radiating or nonradiating chest pain or anginal equivalent & $444(89)$ & $452(91)$ & \\
\hline Arm, jaw, shoulder, or epigastric pain & $21(4)$ & $16(3)$ & \\
\hline Shortness of breath & $7(1)$ & $10(2)$ & \\
\hline Other & $29(6)$ & $21(4)$ & \\
\hline Heart rate - beats/min & $78 \pm 14$ & $77 \pm 14$ & 0.58 \\
\hline \multicolumn{4}{|l|}{ Blood pressure $-\mathrm{mm} \mathrm{Hg}$} \\
\hline Systolic & $144 \pm 23$ & $144 \pm 23$ & 0.80 \\
\hline Diastolic & $83 \pm 13$ & $83 \pm 13$ & 0.94 \\
\hline BMI & $29.4 \pm 5.3$ & $29.1 \pm 4.8$ & 0.41 \\
\hline $\begin{array}{l}\text { Discharge diagnosis after index emergency department visit or hospitalization - } \\
\text { no. (\%) }\end{array}$ & - & & 0.16 \\
\hline Noncardiac chest pain & $426(85)$ & $445(89)$ & \\
\hline Noncoronary cardiac pain & $7(1)$ & $8(2)$ & \\
\hline
\end{tabular}




\begin{tabular}{|c|c|c|c|}
\hline Variable & CCTA $(\mathbf{N}=501)$ & $\begin{array}{c}\text { Standard Evaluation }(\mathrm{N} \\
=499)\end{array}$ & P Value \\
\hline Coronary chest pain not associated with acute coronary syndrome & $25(5)$ & $14(3)$ & \\
\hline Acute coronary syndrome & $43(9)$ & $32(6)$ & \\
\hline Unstable angina pectoris & $35(7)$ & $17(3)$ & \\
\hline Myocardial infarction & $8(2)$ & $15(3)$ & \\
\hline
\end{tabular}

Plus-minus values are means \pm SD. BMI denotes body-mass index (the weight in kilograms divided by the square of the height in meters), and CCTA coronary computed tomographic angiography. 
Table 2

Primary and Secondary Effectiveness and Safety End Points.

\begin{tabular}{|c|c|c|c|}
\hline End Point & CCTA $(N=501)$ & Standard Evaluation $(\mathrm{N}=499)$ & P Value \\
\hline \multicolumn{4}{|l|}{ Length of hospital stay $-\mathrm{hr}$} \\
\hline All patients in intention-to-treat analysis & & & $<0.001$ \\
\hline Mean & $23.2 \pm 37.0$ & $30.8 \pm 28.0$ & \\
\hline Median & 8.6 & 26.7 & \\
\hline Interquartile range & $6.4-27.6$ & $21.4-30.6$ & \\
\hline Patients with final diagnosis other than acute coronary syndrome & & & $<0.001$ \\
\hline Mean & $17.2 \pm 24.6$ & $27.2 \pm 19.5$ & \\
\hline Median & 8.1 & 26.3 & \\
\hline Interquartile range & $6.2-24.6$ & $20.6-29.5$ & \\
\hline Patients with final diagnosis of acute coronary syndrome & & & 0.87 \\
\hline Mean & $86.3 \pm 72.3$ & $83.8 \pm 61.3$ & \\
\hline Median & 56.9 & 71.8 & \\
\hline Interquartile range & $46.2-95.9$ & $45.2-96.7$ & \\
\hline \multicolumn{4}{|l|}{ Time to diagnosis $-\mathrm{hr}$} \\
\hline All patients in intention-to-treat analysis & & & $<0.001$ \\
\hline Mean & $10.4 \pm 12.6$ & $18.7 \pm 11.8$ & \\
\hline Median & 5.8 & 21.0 & \\
\hline Interquartile range & $4.0-9.0$ & $8.5-23.8$ & \\
\hline Patients with final diagnosis other than acute coronary syndrome & & & $<0.001$ \\
\hline Mean & $10.6 \pm 12.3$ & $18.8 \pm 12.0$ & \\
\hline Median & 6.1 & 21.1 & \\
\hline Interquartile range & $4.0-9.6$ & $8.7-23.8$ & \\
\hline Patients with final diagnosis of acute coronary syndrome & & & 0.002 \\
\hline Mean & $8.0 \pm 15.1$ & $17.1 \pm 9.5$ & \\
\hline Median & 4.4 & 14.9 & \\
\hline Interquartile range & $3.3-5.6$ & $7.4-25.1$ & \\
\hline Discharge status - no. $(\%)$ & & & $<0.001$ \\
\hline Direct discharge from emergency department & $233(47)$ & $62(12)$ & \\
\hline Admission to observation unit & $153(30)$ & $301(60)$ & \\
\hline Admission to hospital & $107(21)$ & $125(25)$ & \\
\hline Left against medical advice & $8(2)$ & $11(2)$ & \\
\hline \multicolumn{4}{|l|}{ Follow-up for recurrent chest pain within 28 days - no. } \\
\hline Repeat visit to emergency department & 14 & 19 & 0.38 \\
\hline Repeat hospitalization & 7 & 7 & \\
\hline \multicolumn{4}{|l|}{ Safety - no. } \\
\hline Undetected acute coronary syndrome & 0 & 0 & \\
\hline Periprocedural complications & 2 & 0 & 0.50 \\
\hline
\end{tabular}




\begin{tabular}{|lccc|}
\hline End Point & CCTA $(\mathbf{N}=\mathbf{5 0 1})$ & Standard Evaluation $(\mathbf{N}=499)$ & P Value \\
\hline Length of hospital stay $-\mathbf{h r}$ & & & \\
\hline Major adverse cardiovascular events at 28 days - no. & 2 & 6 & 0.18 \\
\hline
\end{tabular}

Plus-minus values are means \pm SD. 


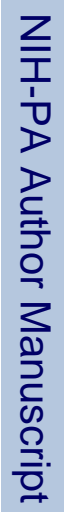

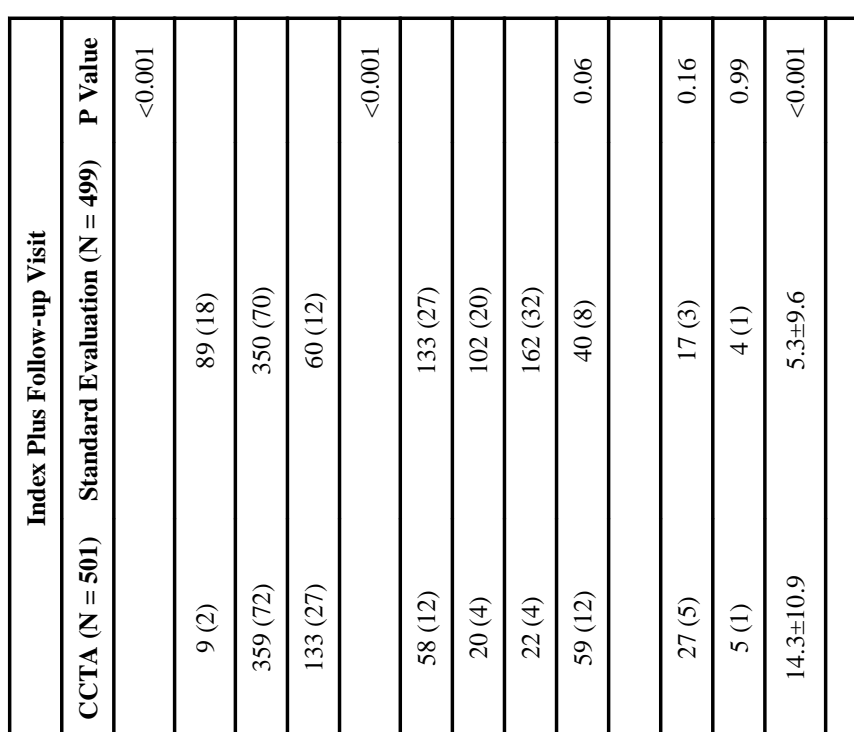

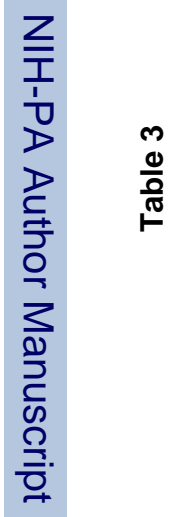

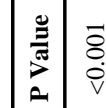

$\overline{8}$
$\dot{\nabla}$
$\dot{v}$

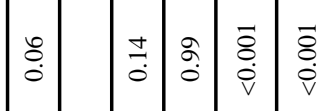

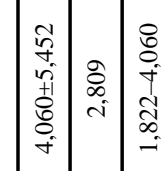

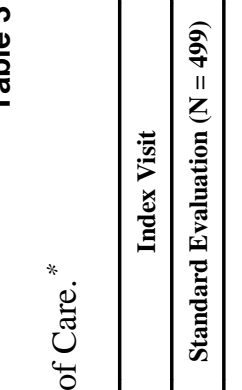

\begin{tabular}{c|c}
0 \\
0 \\
0 \\
0 \\
0 \\
0 \\
$\tilde{\sigma}$ \\
0 \\
0 \\
0
\end{tabular}

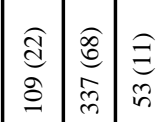

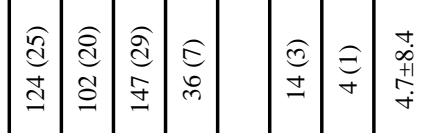

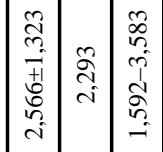

잉

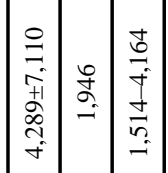

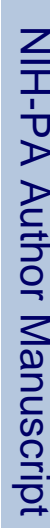

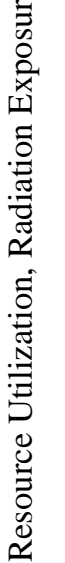

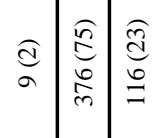

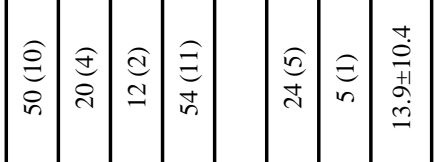

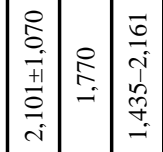

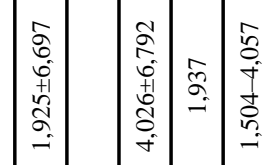

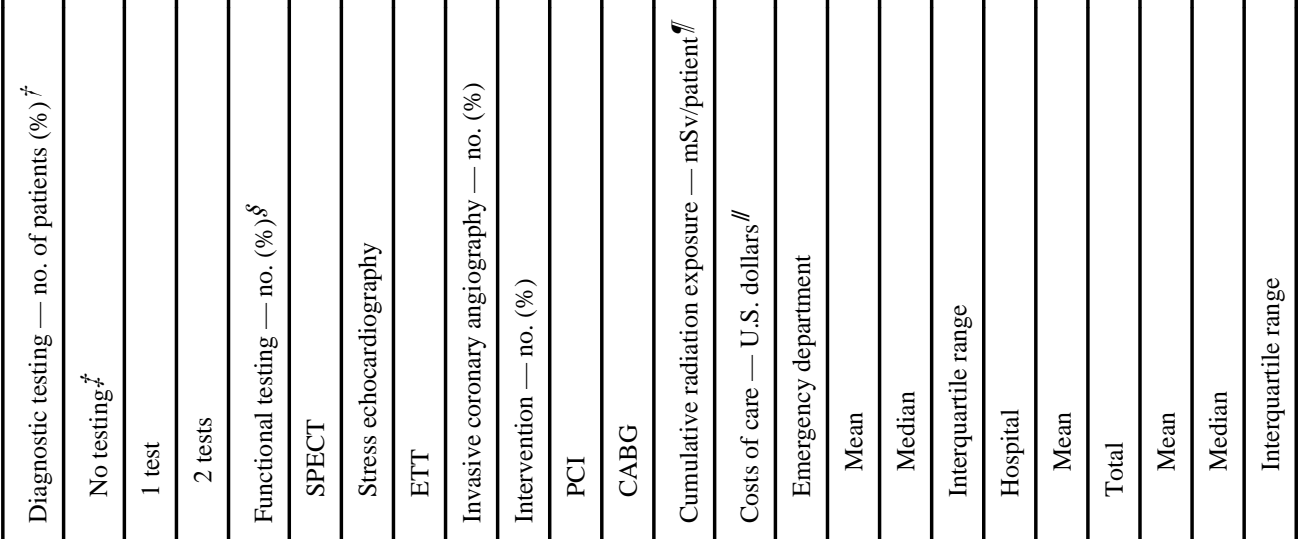

N Engl J Med. Author manuscript; available in PMC 2013 May 23. 


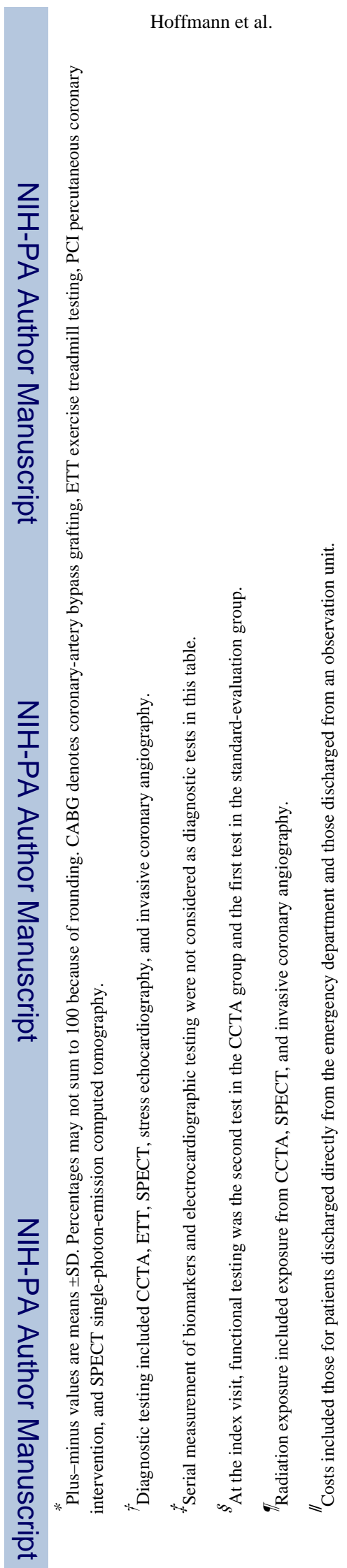

N Engl J Med. Author manuscript; available in PMC 2013 May 23. 